\title{
New method for the production of thin and stable, segmented $n+$ contacts in HPGe detectors
}

\author{
S. Bertoldo ${ }^{1,2,3}$, G. Maggioni ${ }^{1,4, a}{ }^{(\mathbb{D}}$, W. Raniero ${ }^{1}$, C. Carraro $^{1,4}$, S. Riccetto ${ }^{5}$, F. Sgarbossa ${ }^{1,4}$, D. Scarpa ${ }^{1}$, \\ A. Andrighetto ${ }^{1}$, A. Mazzolari ${ }^{6}$, A. Gadea ${ }^{3}$, D. R. Napoli ${ }^{1}$, E. Napolitani ${ }^{1,4}$, D. De Salvador ${ }^{1,4}$ \\ ${ }^{1}$ Laboratori Nazionali di Legnaro, Istituto Nazionale di Fisica Nucleare, Viale dell’Università 2, 35020 Legnaro, PD, Italy \\ ${ }^{2}$ Facultat de Fisica, Universitat de Valencia, C/ Doctor Moliner 50, 46100 Burjassot, Spain \\ ${ }^{3}$ Instituto de Física Corpuscular, C/Catedrático José Beltrán 2, 46980 Paterna, Spain \\ ${ }^{4}$ Dipartimento di Fisica e Astronomia “G. Galilei”, Università di Padova, Via Marzolo 8, 35121 Padua, PD, Italy \\ ${ }^{5}$ INFN, Sezione di Perugia \& Dipartimento di Fisica e Geologia, Università di Perugia, Via A. Pascoli, 06123 Perugia, Italy \\ ${ }^{6}$ INFN Sezione di Ferrara, Via Saragat 1, 44124 Ferrara, Italy
}

Received: 30 November 2020 / Accepted: 11 May 2021 / Published online: 2 June 2021

(C) The Author(s) 2021

Communicated by Wolfram Korten

\begin{abstract}
The realization of pulsed-laser diffused, thin $\mathrm{n}+$ contacts on high purity germanium (HPGe) and their successful segmentation is described. The contacts have been obtained by a laser-induced diffusion of Sb atoms, deposited by sputtering on Ge surface, and then segmented by means of a photolithographic technique. Three small prototypes of gamma ray detectors have been implemented, using the same $\mathrm{n}+$ contact (laser diffused $\mathrm{Sb}$ ) but with three different geometries and a B implanted p+ contact. Electrical and detection properties of the prototypes have been characterized, showing low leakage currents and good spectroscopy data with different gamma-ray sources. The stability of the detector performance has also been tested subjecting one of the prototypes to a typical annealing treatment.
\end{abstract}

\section{Introduction}

Among the different types of radiation detectors, high-purity germanium (HPGe) detectors stand out for their excellent energy resolution and high radiation detection efficiency, which have been making them a well-established and irreplaceable tool in precision gamma-ray spectroscopy, in spite of the need of cryogenic cooling during normal operation. A well-established process for the fabrication of HPGe detectors, based on boron implantation ( $\mathrm{p}+$ contacts) and lithium diffusion ( $\mathrm{n}+$ contacts), has been developed and mature products are nowadays available and commercialized such as the highly segmented detectors used in the latest generation gamma spectrometers like AGATA [1] and GRETA [2]. How-

\footnotetext{
a e-mail: maggioni@lnl.infn.it (corresponding author)
}

ever, in modern applications gamma-ray imaging is often involved and a precise measurement of the interaction position of incident gamma-rays needs to be accomplished. This requirement is pushing the development of detectors, which combine excellent energy resolution with fine position resolution. A key area of development is the creation of electrical contacts, which enable reliable operation, provide low electronic noise, and allow fine segmentation of both electrodes. B-implanted contact can be segmented in a variety of geometries and provides thin dead layers for the gamma-ray detection. Li contact, instead, gives rise to thick dead layers (up to $1 \mathrm{~mm}$ ), it is thermally unstable and can only be coarsely segmented by grinding or using wide inter-segment gaps [3-6]. Moreover, the low dopant stability can easily jeopardize the electrical insulation between the Li segments.

Amorphous-germanium and amorphous-silicon contacts are nowadays the best alternative contact technology, which allows the segmentation of electrodes for position-sensitive detectors, providing contacts which are able to sustain reasonably high electric fields [7-9]. Unfortunately, the leakage currents of these contacts are higher as compared to Li contacts and their thermal stability is still a critical point [10-12], especially in applications that require annealing treatments to recover the detector performance [13].

The current unavailability of a stable and segmentable $\mathrm{n}+$ contact hinders the realization of $\mathrm{p}$-type coaxial segmented detectors to be used in HPGe gamma-ray tracking arrays. This, instead, would be an important progress, since electron collecting segmented detectors would be less sensitive to radiation damage [14] and more prompt to remove the damage and restore the original energy resolution by annealing [15], extending the applicability of gamma track- 
ing arrays under higher damaging hadron flux conditions. Moreover, other applications such as low background experiments (e.g. LEGEND [16] and CDEX [17]) could benefit from using p-type HPGe detectors with thin contacts. At last, another important application would involve doublesided strip detectors, as this type of detectors requires robust and thin $\mathrm{n}+$ and $\mathrm{p}+$ contacts to enable a $3 \mathrm{D}$ position reconstruction of gamma-ray interactions.

Pulsed-laser diffused contacts have a great potential to make new HPGe detector designs easier by providing thin contacts, which can block either electrons or holes by choosing the suitable doping element. Pulsed-laser technique, which exploits laser pulses with high energy density to induce the melting of a surface layer followed by ultra-fast liquid phase epitaxial regrowth, allows to produce highly doped contacts, nearly ideal p-n junctions and recrystallized nearsurface regions, which are free of extended defects $[18,19]$. Moreover, all the Ge bulk remains at a temperature that is very close to the room temperature, thus preventing the thermally induced diffusion of contaminants inside the crystal [18].

In a very recent work [20], we described the successful realization of a thin hole-barrier contact in HPGe by means of a new technique based on the sputter deposition of a $\mathrm{Sb}$ film on Ge surface, followed by pulsed laser annealing, which induces Sb diffusion into Ge. We demonstrated that this technique does not contaminate the hyperpure germanium [21] and keeps the surface free from defects. Moreover, in spite of the very small thickness $(\leq 100 \mathrm{~nm})$ of the hole-barrier contact, the barrier effect is very pronounced as shown by the reverse leakage current, which shows consistently low values [20]. The new method was successfully applied to the realization of small prototypes of planar detectors with the new Sb-based contact on one side and with a standard boron implanted contact on the other side.

In this paper, we describe the successful segmentation of pulsed-laser diffused, thin contacts and its application to the production of three different prototypes of radiation detectors. In order to control the contribution of the leakage current coming from the lateral surface, a guard ring geometry was realized on the $\mathrm{Sb} \mathrm{n}+$ contact side. Inside the guard ring, 1 or 2 insulated contacts were created.

The contact segmentation was obtained by a lithographic procedure on a continuous Sb doped layer. The doped layer was produced through the laser process by scanning the beam over the planar detector surface. The laser-irradiated areas were partially overlapped during the scan in order to avoid undoped regions. To study this procedure, the effect of multiple impulses on the $\mathrm{Sb}$ concentration profile was also investigated. This is particularly important in the perspective of scaling-up the pulsed-laser diffused contacts in order to produce large area highly segmented detectors for gamma-ray spectroscopy and paves the way to the implementation of electron collecting segmented contacts.

Taking into account that several applications of segmented detectors involve important radiation damage to the Ge crystal, with the formation of crystalline defects and charge traps $[1,22,23]$, a key feature of every new contact proposed for these devices is the ability to withstand the thermal treatments (annealing) necessary to remove the radiation damage and recover the detector performance. For this reason, one of the prototypes was subjected to a typical annealing used for radiation damage removal and its performance was tested before and after annealing.

\section{Experimental}

\subsection{Processing of Ge crystals}

Two different Ge crystal wafers (Umicore, Belgium) were used as starting materials for the experiments. Table 1 shows the main properties of each of the two wafers.

Type $1 \mathrm{Ge}$ wafer was cleaved by hand to smaller samples (hereafter referred to as Ge samples), which were used for the characterization of the production process of the $\mathrm{Sb}$ based $n+$ contact. Ge samples were coated with a $\mathrm{Sb}$ film and laser annealed (see below). The characterization of these samples was carried out using Secondary Ion Mass Spectrometry (SIMS), Rutherford Backscattering Spectrometry (RBS) and electrical activation measurements (see below for details).

Type 2 Ge wafer was used to build the radiation detector prototypes. Both faces were manually grinded, then the wafer was cut into $10 \times 10 \mathrm{~mm}^{2}$ samples (hereafter referred to as HPGe samples) using an automatic dicing machine (Disco Corporation, Tokyo, Japan). The HPGe samples were cleaned with hot 2-propanol, hot deionized (DI) water and HF $10 \%$ to remove the dicing adhesive residue and the native oxides. The square HPGe samples were then chemically etched for 5 to 7 minutes using a $3: 1 \mathrm{HNO}_{3} 65 \%$ : $\mathrm{HF} 40 \%$ solution (all acids were of reagent grade, Carlo Erba Reagents srl) in order to remove the mechanically damaged surface layer. Chemical etching caused a decrease of the substrate thickness down to $1.95 \pm 0.05 \mathrm{~mm}$. After this step HPGe samples underwent boron implantation $\left(23 \mathrm{keV}, 1 \times 10^{15}\right.$ atoms $\mathrm{cm}^{-2}$ ) on one side in order to obtain the electronbarrier contact (B contact), before proceeding with the deposition of a 2-nm-thick $\mathrm{Sb}$ film, which was carried out on the whole surface on the other side of the samples. After the laser annealing of the Sb-coated face and the deposition of Au electrical contacts, the junction properties were tested by measuring the leakage current as a function of the reverse polarization potential (see below). 
Table 1 Properties of Ge starting materials (notes: $\mathrm{CMP}=$ chemi$\mathrm{cal} /$ mechanical polish; as-cut $=$ after the saw cut; n.a. $=$ not available $; *$ = impurity concentration and resistivity was previously measured [21] and coincided with the value given by Umicore. It should be noted that both wafers are intrinsic at room temperature with hole and electron intrinsic carrier concentration of $2 \times 10^{13} \mathrm{~cm}^{-3}$ [24] and a resistivity of $40 \Omega \mathrm{cm}$ )

\begin{tabular}{|c|c|c|c|c|c|c|c|c|}
\hline Ge wafer & $\begin{array}{l}\text { Crystal } \\
\text { growth } \\
\text { axis }\end{array}$ & Carrier type & $\begin{array}{l}\text { Thickness } \\
(\mathrm{mm})\end{array}$ & $\begin{array}{l}\text { Diameter } \\
(\mathrm{mm})\end{array}$ & Surface finish & $\begin{array}{l}\text { Impurity concen- } \\
\text { tration }\left(\mathrm{cm}^{-3}\right)\end{array}$ & $\begin{array}{l}\text { Resistivity at } \\
25^{\circ} \mathrm{C}(\Omega \mathrm{cm})\end{array}$ & Usage \\
\hline 1 & $(100)$ & $\mathrm{n}$ & 0.5 & 100 & CMP & n.a. & 40 & $\begin{array}{l}\text { SIMS, RBS, electrical } \\
\text { activation }\end{array}$ \\
\hline 2 & (100) & $\mathrm{p}$ & 2. & 50 & As-cut & $10^{10 *}$ & $40^{*}$ & $\begin{array}{l}\text { Detector processing } \\
\text { and test }\end{array}$ \\
\hline
\end{tabular}

Sb layers were deposited on Ge and HPGe samples by sputtering using an equipment consisting of a stainless-steel vacuum chamber evacuated by a turbomolecular pump at a base pressure lower than $1 \times 10^{-4} \mathrm{~Pa}$. The chamber was equipped with a cylindrical magnetron sputtering source, connected to a radio frequency power generator (Advanced Energy, $600 \mathrm{~W}, 13.56 \mathrm{MHz}$ ) through a matching box. The deposition parameters used for all the films were: target-tosubstrate distance $14 \mathrm{~cm}$; working gas $\operatorname{Ar}$ (99.9999\% purity); Ar flow $3.33 \times 10^{-7}$ standard $\mathrm{m}^{3} \mathrm{~s}^{-1}$; direct RF power $30 \mathrm{~W}$. Pure $\mathrm{Sb}$ (99.999\%, ACI Alloys) with a very low $\mathrm{Cu}$ content $(\leq 0.1 \mathrm{mg} / \mathrm{kg}$ ) was used as target. The deposition rate was determined by RBS and the duration of the deposition run was fixed in order to achieve the desired film thickness.

A pulsed Nd:YAG solid state laser, characterized by $7 \mathrm{~ns}$ pulse duration and $10 \mathrm{~Hz}$ repetition rate, was used for the $\mathrm{Sb}$ diffusion. The laser light was in the UV range $(355 \mathrm{~nm})$ and the energy density deposited on the sample during the exposure to a single pulse was $400 \mathrm{~mJ} \mathrm{~cm}^{-2}$.

Three Sb-coated HPGe samples were laser irradiated using the geometry depicted in Fig. 1a. The geometry consists in nine partially-overlapped $4 \times 4 \mathrm{~mm}^{2}$ square spots (only four spots are shown in the figure, three along the diagonal of the square sample and one with dashed lines for clarity). The partial overlap between two contiguous spots is $0.7 \mathrm{~mm}$ in both directions and the lateral spots protrude $0.3 \mathrm{~mm}$ from the edges of the sample. The surface areas exposed to 2 laser pulses (blue rectangles) and 4 laser pulses (red squares) are also highlighted.

After laser annealing, the Sb-doped surface was cleaned with hot 2-propanol, hot deionized (DI) water and HF $10 \%$ and then two different processes were used for the Au electrode deposition and contact segmentation for the three HPGe samples:

-sample A: a circular Au electrode with a diameter of 5 $\mathrm{mm}$ and an external guard ring ( $1 \mathrm{~mm}$ gap in between) was deposited by sputtering (Fig. 1b). Au film was $100 \mathrm{~nm}$ thick

sample B1: a 100-nm-thick Au film was deposited on the whole $\mathrm{Sb}$-doped surface and then a lithographic process was used to create the guard ring and two contacts inside (Fig. 1c). The steps of the lithographic process were:

- dripping $0.3 \mathrm{ml}$ of positive photoresist (Microposit S1813) on top of the Au-coated surface;

- spin coating of photoresist (500 rpm for $5 \mathrm{~s}$ and $4000 \mathrm{rpm}$ for $40 \mathrm{~s}$ with an acceleration of $4000 \mathrm{rpm} / \mathrm{s})$;

- soft baking $\left(130^{\circ} \mathrm{C}\right.$ for 1 minute);

- UV irradiation (UVA 400W lamp at 350-400 nm wavelength) for $50 \mathrm{~s}$ through an acetate mask with $10 \mu \mathrm{m}$ resolution. The mask allows obtaining a geometry consisting in two segments (area $2.4 \times 5.6 \mathrm{~mm}^{2}$ each segment) separated by a $0.4 \mathrm{~mm}$ gap and surrounded by a guard ring (0.4 mm gap between segments and guard ring);

- removal of the irradiated part of the resist with a proper solvent (Tetramethylammonium hydroxide, TMAOH);

- long soft baking $\left(130^{\circ} \mathrm{C}\right.$ for 15 minutes);

- removal of the unwanted $\mathrm{Au}$ with a gold etchant $\left(\mathrm{KI} / \mathrm{I}_{2}\right.$, MicroChemicals);

- removal of the remaining resist with hot acetone;

- cleaning with hot 2-propanol and hot DI water;

- cleaning in TMAOH, gold etchant and acetone baths for three times with hot DI water rinsing in between to avoid material deposition on the HPGe surface.

-sample B2: the same process as sample B1 with the only difference in the size of the gap between segments $(0.2 \mathrm{~mm}$ instead of $0.4 \mathrm{~mm}$ ).

For all the three HPGe samples the removal of the Sbdoped layer from the gaps and the passivation of all the intrinsic surfaces were done in a single step by etching for $10 \mathrm{~s}$ in a $3: 1 \mathrm{HNO}_{3} 65 \%$ : HF $40 \%$ acid solution followed by quenching in methanol (Erbatron, Carlo Erba) [25]. As previously shown [26], this passivation procedure is based on the dissociative adsorption of methanol on the Ge surface, which leads to $\mathrm{Ge}-\mathrm{H}$ and $\mathrm{Ge}-\mathrm{O}-\mathrm{CH}_{3}$ covalent bonds. The samples were then dried with a nitrogen blow. During this etching, the electron-barrier contact (B contact) was protected by Kapton tape. 
Fig. 1 a Laser irradiation geometry in $\mathrm{mm}$ (see text for details). b Photo of a square HPGe prototype (sample A) showing the Sb-doped face with Au circular electrode and guard ring. c Photo of a square HPGe prototype (sample B1) showing the $\mathrm{Sb}$-doped face with $\mathrm{Au}$ electrodes ( $\mathrm{S} 1$ and $\mathrm{S} 2$ segments, $0.4 \mathrm{~mm}$ gap) and guard ring

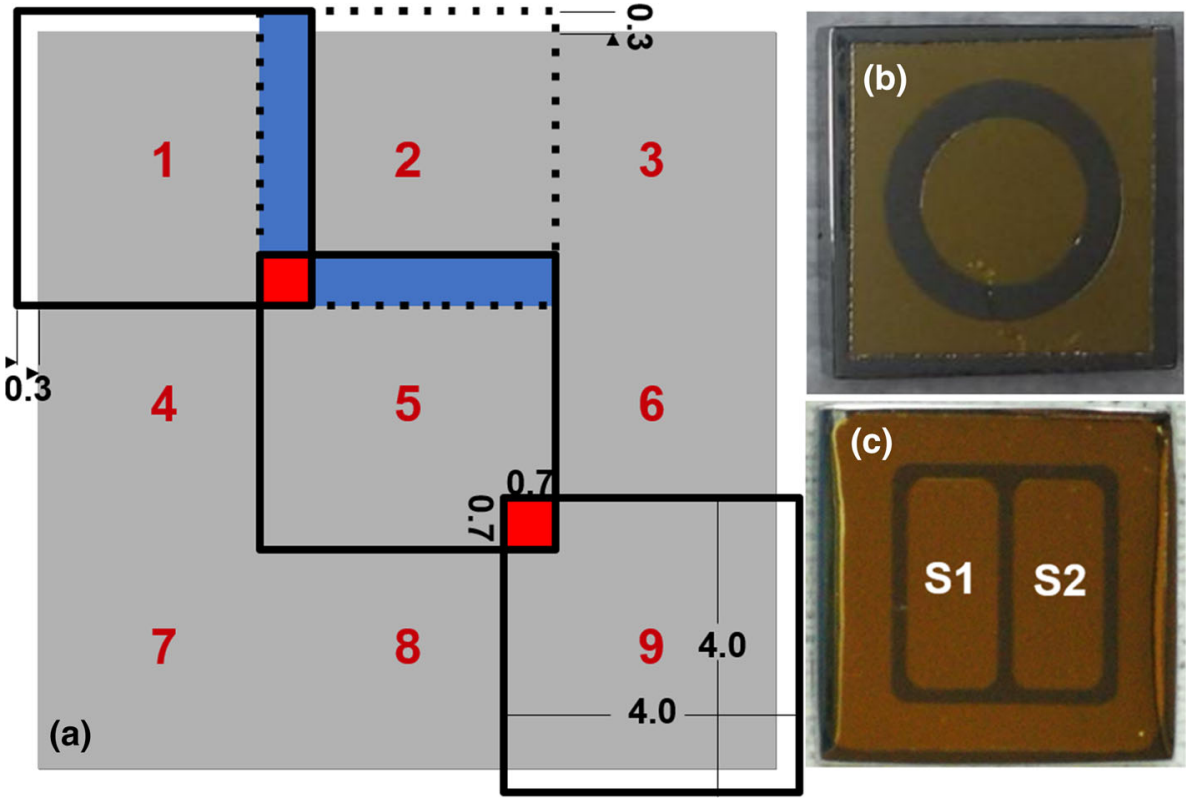

\subsection{Characterization of Ge samples (wafer 1)}

Secondary Ion Mass Spectrometry (SIMS) was performed on laser annealed samples, by using a Cameca IMS-4f instrument with an $\mathrm{O}_{2}^{+}$beam, to characterize the $\mathrm{Sb}$ diffusion profiles. Rutherford Backscattering Spectrometry (RBS) was carried out using $2.0 \mathrm{MeV}^{4} \mathrm{He}^{+}$beam at the Van de Graaff accelerator at the INFN Laboratori Nazionali di Legnaro with a $160^{\circ}$ scattering angle.

The measurement of the electrical activation of diffused Sb was performed through the Van der Pauw-Hall technique, using a 4-point probe apparatus (4PP). This instrument provides the doped layer sheet resistance $\mathrm{R}_{\mathrm{S}}$ and, given the application of a magnetic field ( $0.625 \mathrm{~T}$ using a permanent magnet), the Hall coefficient $\mathrm{RH}_{\mathrm{S}}$ [27]. These values must be carefully analysed considering that the carrier profile is not uniform and that both mobility and resistivity are a function of the carrier concentration. Therefore, the values resulting from 4PP measurements must be interpreted as weighted averages as previously discussed in literature [28,29]. A more detailed procedure for this analysis is provided in the aforementioned study [28].

\subsection{Characterization of HPGe samples (wafer 2)}

For the measurements of the leakage current, the first detector sample (sample A) was mounted on a commercial standard cryostat (Figure 1 of Ref. [20]) and cooled to $(91 \pm 1) \mathrm{K}$. A $1.0 \mathrm{~mm}$ thick Indium foil was inserted between each side of the detector and the metal supports, in order to improve the electrical contact. A source/measure unit (SMU, Keithley
237, $0.1 \mathrm{pA}$ resolution) was connected to both contacts and guard ring and used for the measurements of the $\mathrm{I}-\mathrm{V}$ curves.

The two detector samples B1 and B2 were mounted one at a time on a prototype cryostat developed for segmented detectors and cooled to $(110 \pm 2) \mathrm{K}$. Metal springs mounted on a support ensured electrical contact on the segmented side while a $1.0 \mathrm{~mm}$ thick Indium foil was inserted between the non-segmented side and the polarizing metal support. In this setup, a SMU (Keithley 237, 0.1 pA resolution) was connected to the $\mathrm{p}+$ side to polarize the detector; a second SMU (Keithley 237, $0.01 \mathrm{pA}$ resolution) measured the current on one segment while the other segment and the guard ring were connected to the cryostat chassis.

The experimental setup used for the measurement of the electrical resistance between the segments of detector B1 (intergap resistance) is sketched in Fig. 2. Three SMUs were used for these measurements: two SMUs (SMU1 and 2, Keithley $237,0.01 \mathrm{pA}$ and $0.1 \mathrm{pA}$ resolution, respectively) were connected to the segments. SMU1 kept the potential on the first segment at $0 \mathrm{~V}$ and SMU2 polarized the second segment at $\Delta \mathrm{V}$. With this configuration, the former segment is at the same potential as the guard ring, which is directly connected to ground, so that the only currents that can reach this electrode come from the second polarized segment or by a bulk leakage from the $\mathrm{p}+$ contact. In order to reduce this bulk leakage, and to be more sensitive to surface conductivity, the third SMU (SMU3, Keithley 2400, 10 pA resolution) was connected to the $\mathrm{p}+$ contact and kept it at a negative reverse potential. At the applied potential $\mathrm{V}=-12 \mathrm{~V}$, the leakage is always negligible and the depletion region can be estimated to be $\mathrm{W}=1.4 \mathrm{~mm}$, according to the formula [30]:

$\mathrm{W}=(2 \varepsilon \mathrm{V} / \mathrm{eN})^{1 / 2}$ 


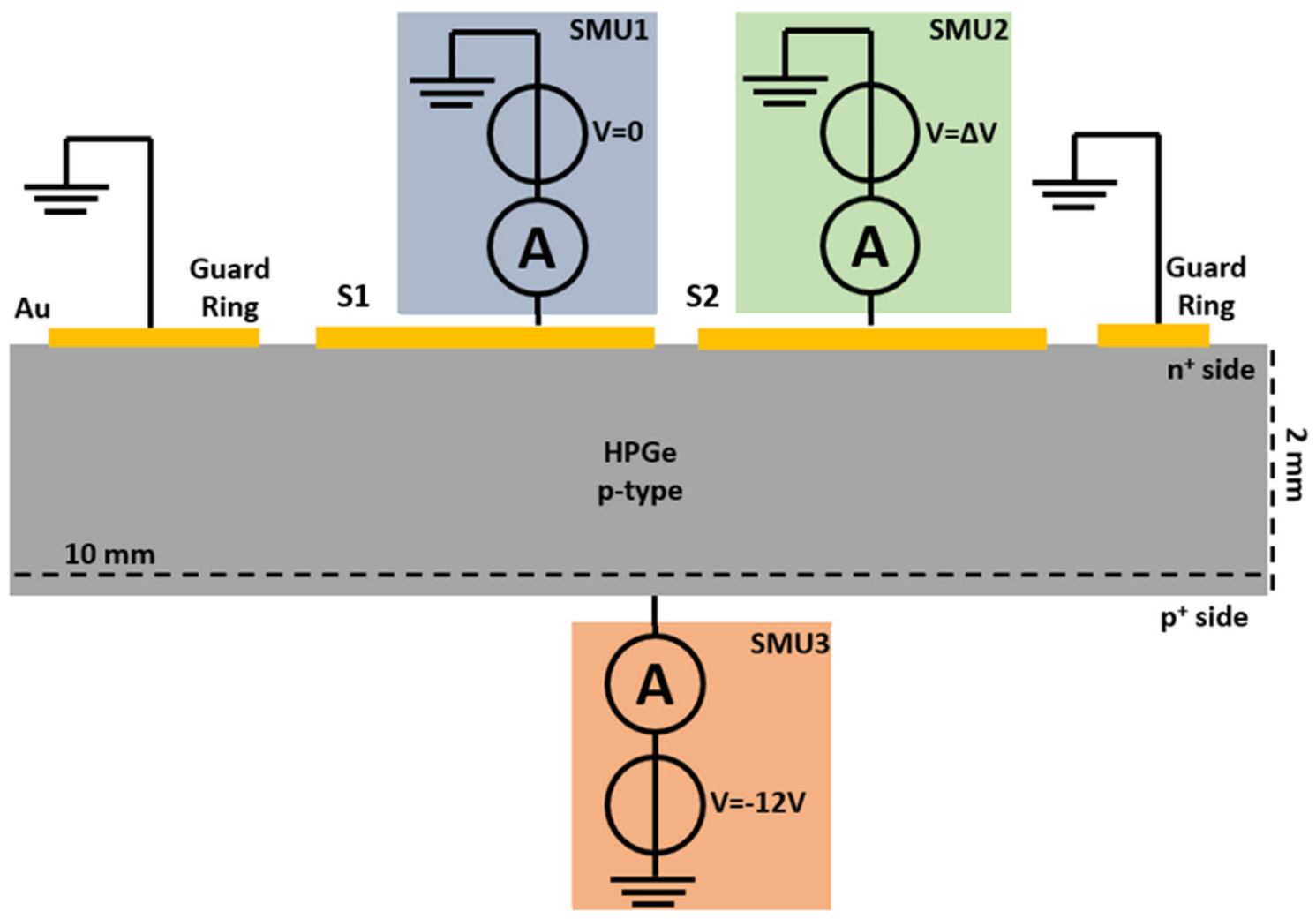

Fig. 2 Sketch of the electrical circuit used for the measurements of the electrical resistance of the inter-segment gap: a cross section of the detector shown in Fig 1c represents the device under test

where $\varepsilon$ is the dielectric constant of germanium, e the electronic charge and $\mathrm{N}=1 \times 10^{10} \mathrm{~cm}^{-3}$ is the bulk impurity concentration. This configuration allows to measure the resistance between the two segments as the ratio between $\Delta \mathrm{V}$ and the current on SMU1. For completeness, the same configuration was applied for both segments simply swapping their roles.

The same cryostats were used for the determination of the detector properties. Standard calibrated gamma-ray sources of ${ }^{241} \mathrm{Am}$ and ${ }^{133} \mathrm{Ba}$ were used for the detector tests. For detector A, the sources were placed as close as possible to the lateral side of the detector in order to minimize the absorbing material thickness between source and detector, i.e. $1 \mathrm{~mm} \mathrm{Al}$ for the mounting cap and $1 \mathrm{~mm} \mathrm{Al}$ for the external end cap. The different configuration of the cryostat prototype allowed instead the frontal positioning of the sources for detectors B1 and B2, even if a $20-\mu$-thick brass sheet was put between detector and sources. Detector properties were measured by using the ${ }^{241} \mathrm{Am}$ source, while the ${ }^{133} \mathrm{Ba}$ source was used for the energy calibration. Analog NIM electronics was used for the detector measurements: a preamplifier (ORTEC model 257 for detector A and AGATA-type triple segment preamplifier developed by the University of Milano for detectors B1 and B2), an ORTEC spectroscopy amplifier (model 672) with $6 \mu$ s shaping time, a Keithley 2400 SMU and an ORTEC
Easy-MCA 8K with Maestro software. The $6 \mu$ s shaping time was chosen after tests on detectors of similar thickness and area and gave the best energy resolution [20].

\section{Results and discussion}

\subsection{Diffusion and electrical activation}

In a previous work [20] we measured the SIMS chemical concentration profile of antimony diffused in germanium after annealing treatment with a single laser pulse. This measurement pointed out that the $\mathrm{Sb}$ diffusion was limited to a very thin $(<100 \mathrm{~nm})$ surface layer and that the average $\mathrm{Sb}$ concentration in the doped layer was high (around $10^{21}$ atoms $\mathrm{cm}^{-3}$ ). As observed in the experimental section, in the present work we adopted a new laser irradiation geometry for the detector prototypes, which involves the partial overlap of contiguous, laser-irradiated areas. These overlapping areas are exposed to more than a single laser pulse, and precisely to 2 and 4 pulses (see Fig. 1). Since each impulse produces melting, Sb diffusion and regrowth of the surface layer, multiple impulses lead to changes in the Sb diffusion profile. Therefore, we decided to expose on purpose Sb-coated Ge samples to several consecutive laser pulses and to measure 


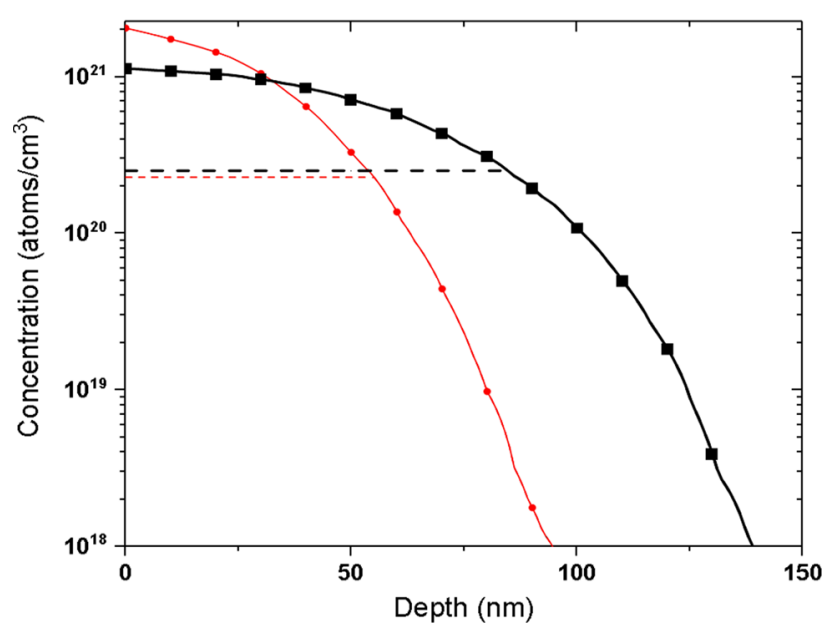

Fig. 3 SIMS chemical concentration profiles of Sb (continuous lines) and electrically active $\mathrm{Sb}$ (dashed lines) diffused in germanium after laser annealing with 1 pulse (red thin lines with dots) and 4 pulses (black bold lines with squares)

the resulting $\mathrm{Sb}$ profiles. A detailed characterization of the results of this study, including a number of impulses from 1 to 8 and a Sb film thickness from one monolayer to $8 \mathrm{~nm}$, can be found in a recent work [28]. Here only the 4-pulses-lasered sample will be considered.

Figure 3 shows the SIMS measurement of this sample as compared to the 1-pulse-lasered one. The depth scale was calibrated measuring the crater depths with a stylus profilometer and assuming constant sputtering rate, while the chemical concentration calibration was performed using a suitable standard. As it can be seen, the main effect of the 4 impulses is the flattening of the $\mathrm{Sb}$ profile (continuous lines), consisting in a slight decrease of the dopant concentration close to the surface and in an increase in depth with an elongation of the diffusion tail. Nevertheless, the thickness of the doped layer is still very low $(<150 \mathrm{~nm})$ and the doping atoms stay close to Ge surface. It is also noteworthy that neither Sb outdiffusion from Ge nor surface segregation are found after 4 laser pulses. Moreover, Sb surface concentration is higher than $10^{21} \mathrm{~cm}^{-3}$, well above the known solid solubility limit of $\mathrm{Sb}$ in $\mathrm{Ge}\left(1.2 \times 10^{19} \mathrm{~cm}^{-3}\right.$ [31]). The same samples were also tested with the van der Pauw-Hall method in order to gain insight on the electrical activation of diffused $\mathrm{Sb}$. This analysis points to carrier profiles, which follow the SIMS chemical ones, and have a plateau-like structure in the case of partially active dopant, with a maximum active concentration. In Figure 3 carrier depth profiles for both samples (dashed lines) are compared to the corresponding chemical profiles: the top part of the profile is only partially active with a plateaulike structure, while the in-depth tail is fully active. It is noteworthy that the remarkable maximum carrier concentration (about $2.5 \times 10^{20} \mathrm{~cm}^{-3}$ ) is preserved even after 4 laser pulses.

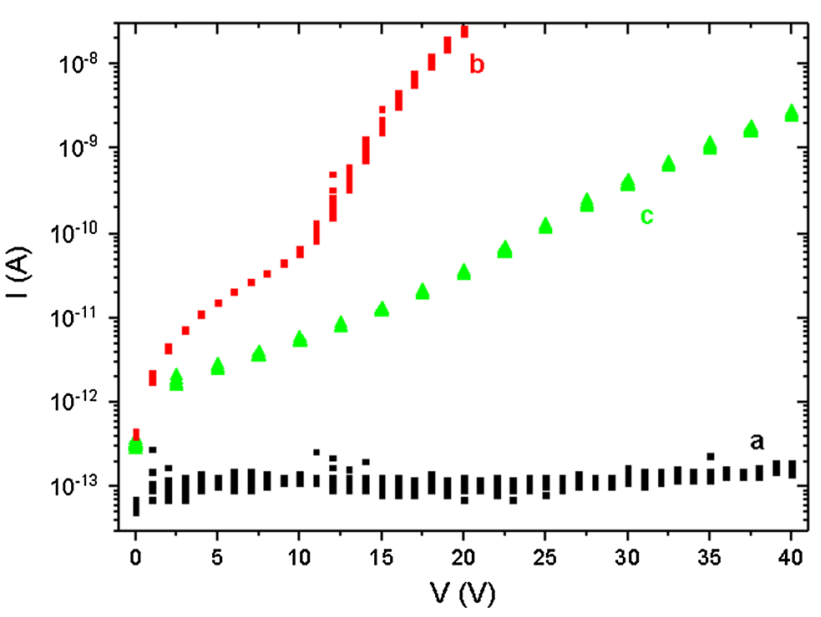

Fig. 4 I-V curves of the detector prototypes: a current between $\mathrm{Sb}$ central contact and B contact (prototype A); $\mathbf{b}$ current between Sb guard ring and $\mathrm{B}$ contact (prototype $\mathrm{A}$ ); $\mathbf{c}$ current between $\mathrm{Sb}$ contact and $\mathrm{B}$ contact for the prototype characterized in Ref. [20]

\subsection{Detector prototypes}

Figure 4 shows the results of the measurements of the reverse leakage current of the detector prototype A (sample with the single central electrode). In the same figure, the leakage current measured for the prototype characterized in Ref. [20] is also shown (curve $\mathrm{c}$ ) in order to highlight the contribution of the surface current: the two prototypes have similar geometry and the only difference is the presence of the guard ring in prototype A. The lack of a guard ring in the prototype of Ref. [20] makes the intrinsic surface passivation particularly critical and, as a final outcome, the leakage current increases up to $300 \mathrm{pA}$ at $40 \mathrm{~V}$. On the other hand, when the guard ring is present, the surface contribution is completely removed: while the current in the guard ring (curve b in Fig. 4) increases considerably, reaching $30 \mathrm{nA}$ at $20 \mathrm{~V}$, the current in the central contact (curve a) keeps well below $1 \mathrm{pA}$ up to $40 \mathrm{~V}$. This very low value of reverse current highlights the remarkable hole barrier properties of the new Sb-doped contact.

I-V curves for prototype B1 $(0.4 \mathrm{~mm}$ gap between the segments S1 and S2) are shown in Fig. 5. The reported data are the S1, S2 and guard-ring currents. We can observe that while the guard-ring current rapidly increases, the currents of both segments are below $0.2 \mathrm{pA}$ in the whole measurement range, demonstrating the high barrier efficiency for both segments.

In order to determine the resistive coupling between the two segments, we used the measurement configuration described in the Experimental section (Fig. 2): the p+ contact of the detector was polarized to $-12 \mathrm{~V}$ in order to deplete the volume close to the junctions (both segments and guard ring) up to a depth of about $1.4 \mathrm{~mm}$. Then, after applying a voltage difference $\Delta \mathrm{V}$ between the segments, the current on either segment was measured. The current measurements 


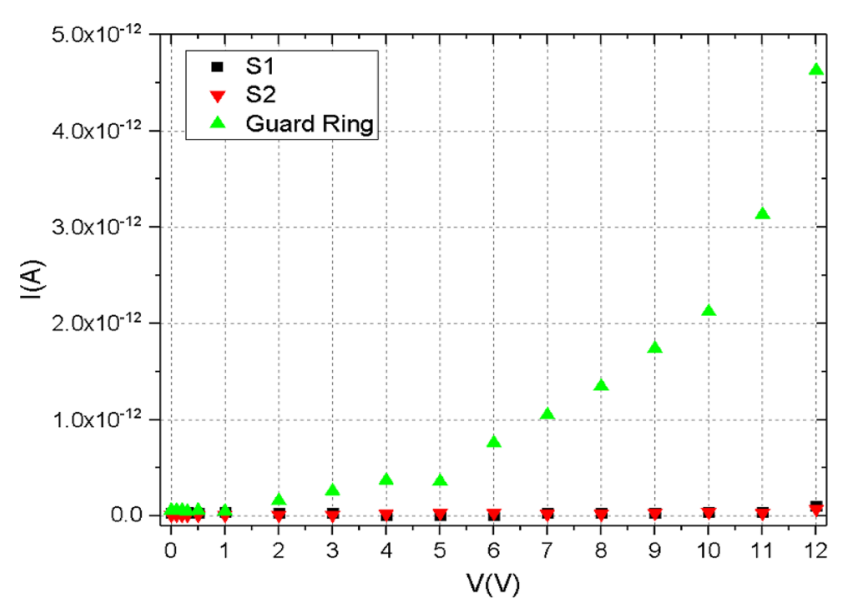

Fig. 5 I-V curves of the detector prototype B1 measured in the diode configuration. S1 and S2 are the two segments of the detector

were carried out by polarizing at $\Delta \mathrm{V} \leq 1 \mathrm{~V}$ : these values are low enough not to disturb the electric field inside the germanium bulk and, in particular, the charge depletion in the volume close to the gap. This means that the contribution of the bulk carriers to the measured current is negligible as compared to that of the surface carriers. For all the $\Delta \mathrm{V}$ values, the gap resistance remains around $1 \mathrm{~T} \Omega$ : this very high value implies that the resistive coupling is negligible, and the dominant contribution is the capacitive coupling, which depends on the geometry of the contacts and of the cryostat.

In order to analyse the spectroscopic properties of the detector prototypes, calibrated gamma-ray sources were used. Figure 6 shows counting rate and energy resolution (FWHM) of prototypes A and B1 as a function of the applied bias voltage for the ${ }^{241} \mathrm{Am}$ photopeak $(\mathrm{E}=59.54 \mathrm{keV})$. Counting rate was determined from the calculation of the photopeak integral (after background subtraction) normalized to the acquisition live time.

As it can be seen in Fig. 6, at first the counting rate is rising with the bias voltage: this trend is due to the increase of the depleted volume in the detector. Afterwards the counting rate reaches a saturation plateau when the detector volume is completely depleted. In agreement with refs. [32,33], the counting rate curve can be used to determine the depletion voltage of a detector, which corresponds to the applied bias voltage at which the counting rate reaches the plateau. Due to the error in the determination of the counting rate, the depletion voltage for the two prototypes is between 15 and $22 \mathrm{~V}$ : this range of values is compatible with the theoretical depletion voltage expected from the impurity concentration in the detector bulk. These values confirm that the laser-based contact technology does not introduce contaminants and preserves the high purity of Ge [21]. A comparison between the plateau values of the counting rates of the two detectors shows that this value is lower for detector A with respect to those of

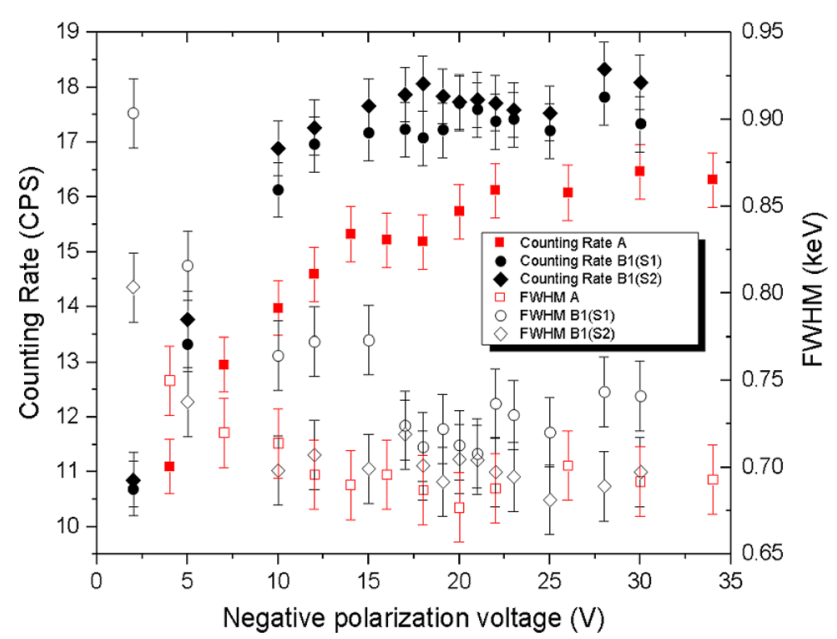

Fig. 6 Counting rate (closed symbols) and FWHM (open symbols) of the ${ }^{241} \mathrm{Am} 59.5 \mathrm{keV}$ photopeak for detector A (red square points) and for the two segments (S1 and S2) in the detector B1 (black points)

the two segments of detector B1, which, on the other hand, are comparable. This difference is due to the different position of the ${ }^{241} \mathrm{Am}$ source (frontal instead of lateral) and to the different cryostats used for the measurements. The energy resolution improves at increasing voltages up to an optimal value $(0.68-0.74 \mathrm{keV})$ around the depletion voltage and then remains constant at higher voltages.

\subsection{Segmentation stability after thermal annealing}

In order to study the effects of a typical annealing used to remove radiation damage and recover detector performance $[13,34]$, we thermally treated prototype $\mathrm{B} 1$ for $40 \mathrm{~h}$ at $105^{\circ} \mathrm{C}$ in a dynamic vacuum $\left(\leq 10^{-3} \mathrm{~Pa}\right.$, under continuous pumping). It should also be noted that the detector was stored at room temperature for a total time of 390 hours before the I-V measurements after annealing. Since it is known that the annealing treatment destroys the surface passivation obtained with chemical etching followed by methanol quenching, the prototype was subjected to a new, short-term passivation treatment ( $5 \mathrm{~s}$ in the acid bath) before proceeding with the required tests. After this re-passivation, both diode and detector performance were measured.

Figure 7 shows I-V curves for both segments and guard ring as measured after annealing and re-passivation. The first important result is that annealing does not affect the barrier properties of the $\mathrm{Sb}$ contact: the current of both segments remains constant and very low (below $0.4 \mathrm{pA}$ ) up to the highest voltage values and in any case well above the depletion voltage. Considering that, to the best of our knowledge, the only other thin $\mathrm{n}+$ contact used in segmented detectors deteriorates its performance after just one annealing treatment [13], this result is particularly important for future applications of $\mathrm{Sb}$ contact. 


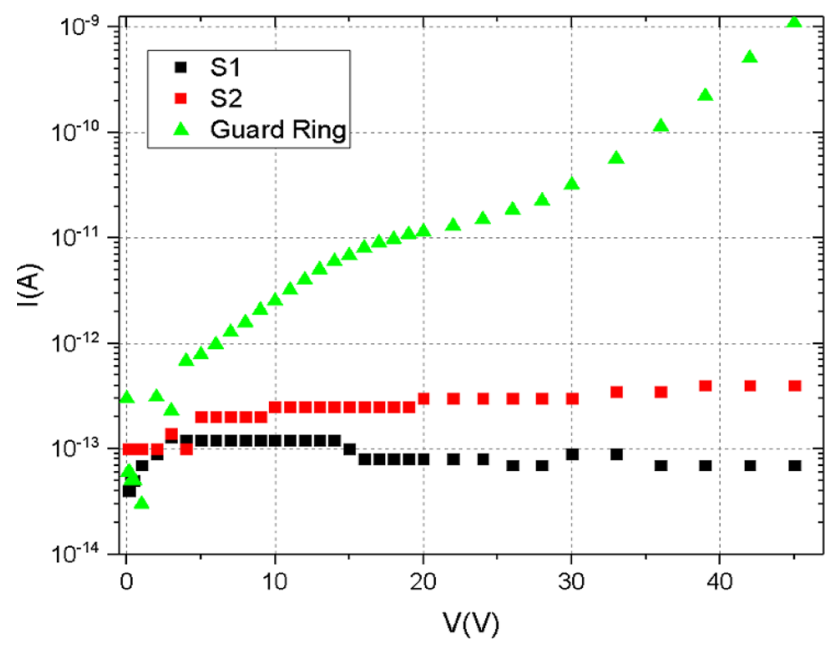

Fig. 7 I-V curves for both segments S1 and S2 and guard ring of prototype $\mathrm{B} 1$ after the annealing process

The electrical insulation between the two segments has also been tested after annealing. It is found that the electrical resistance is still very high $(>10 \mathrm{G} \Omega)$, showing that the electrical insulation is preserved after annealing. It should be noted that the decrease of two orders of magnitude of the resistance after annealing must not be ascribed to the $\mathrm{Sb}$ contact, but to the specific nature of the passivation process chosen for these prototypes, which involves the formation and preservation of a single monolayer on the Ge surface. In both cases, before and after annealing, the resistive coupling between the contacts will have negligible effects with respect to usual capacitance coupling.

Preservation of barrier properties of Sb junction guarantees the correct operation of the annealed detector: Figure 8 shows the total counting rate of prototype $\mathrm{B} 1$ (obtained by adding the counting rates of the two segments in order to reduce source positioning errors) as a function of polarization voltage. As it can be seen, the trend of the counting rate before and after annealing is comparable: in particular, the depletion voltage remains in the same range. This result is particularly important, because it shows that the annealing does not induce contaminant elements in the detector volume that might be introduced just below the surface by laser process and/or other steps of the detector processing and then diffuse even at very low temperatures. Taking into account the low thickness of this prototype $(\leq 2 \mathrm{~mm})$, which makes it more susceptible to the effects of bulk contamination, this result represents also a promising first step in the application of this technology to the realization of thicker and more complex detectors.

Concerning the energy resolution, there are no significant changes after annealing for segment S2. On the other hand, the resolution of segment $\mathrm{S} 1$ improves after annealing and becomes comparable to that of S2: this change is ascribed to

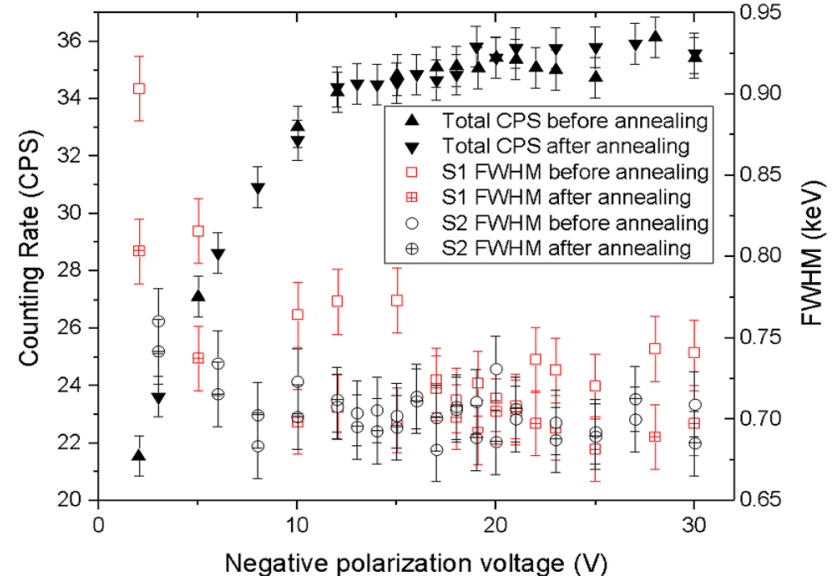

Fig. 8 Total counting rate (closed symbols) and FWHM of the ${ }^{241} \mathrm{Am}$ $59.5 \mathrm{keV}$ photopeak in prototype B1 before annealing (open symbols) and after annealing (crossed symbols)

the presence of traps (structural defects), which are removed by annealing.

\subsection{Inter-segment gap reduction}

The spatial resolution of a segmented detector can be improved by narrowing the inter-segment gap. A further reason for gap reduction is that the narrower the gap, the smaller the underlying dead region, which can degrade the detector performance [35]. In order to explore the potential of the new segmentation technology described in this work, a prototype with a halved gap $(0.2 \mathrm{~mm})$ was then made and first tests were carried out.

$\mathrm{I}-\mathrm{V}$ measurements of this prototype show that the leakage current in the segments remains constantly low, up to voltages higher than $30 \mathrm{~V}$. The trend of the counting rate as a function of bias voltage is comparable to that of prototype $\mathrm{B} 1$ and the depletion voltage is in the range 19-22 V.

Gap reduction did not even affect the energy resolution: Figure 9 shows a comparison between the spectra of ${ }^{241} \mathrm{Am}$ and ${ }^{133} \mathrm{Ba}$ sources collected by one segment of each of the two prototypes. It is worthy to note that the energy resolution is less than $1 \mathrm{keV}$ for all measured energies (below $100 \mathrm{keV}$ ).

Moreover, since both spectra of ${ }^{133} \mathrm{Ba}$ show that the peaks at $30 \mathrm{keV}$ and $35 \mathrm{keV}$ are clearly detectable, it can be stated that the requirement of low-energy threshold can be met, in principle, with this technology.

\section{Conclusions}

We described a new technology for the production of thin segmented $\mathrm{n}+$ contacts in high purity germanium, consisting in the deposition of a Sb layer by sputtering, followed by $\mathrm{Sb}$ diffusion and activation in HPGe by laser annealing 

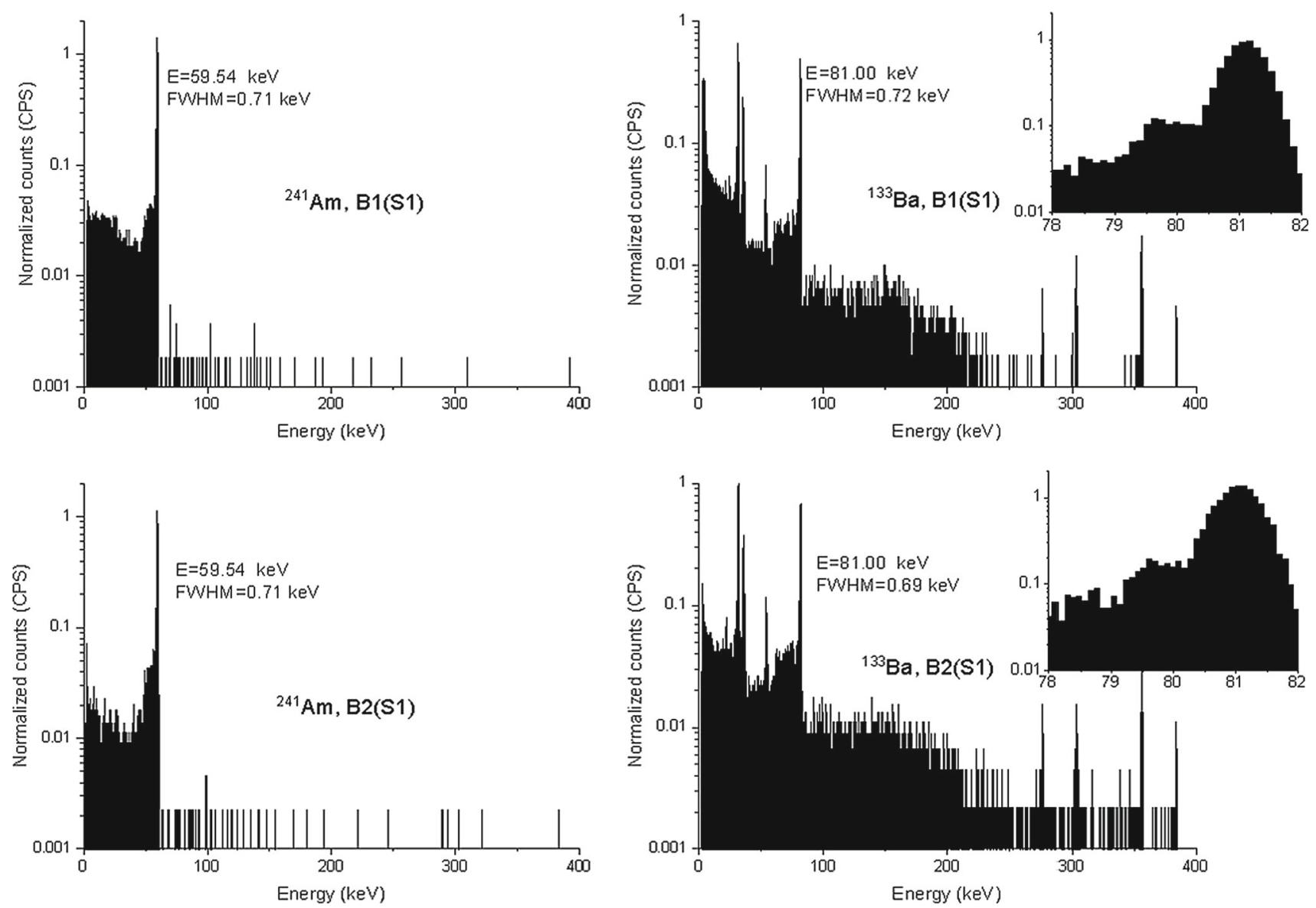

Fig. 9 Spectra normalized to the acquisition time of ${ }^{241} \mathrm{Am}$ and ${ }^{133} \mathrm{Ba}$ sources collected by one segment (S1) of both prototypes B1 (upper spectra) and B2 (lower spectra): insets show the zoomed region around

the $81 \mathrm{keV}{ }^{133} \mathrm{Ba}$ peak after a 2-channels rebinning. The low intensity peak at $79.6 \mathrm{KeV}$ can also be observed

The results of this work are an important step to apply and contact segmentation by a photolithographic technique. The new method was successfully applied to the realization of three detector prototypes. This work represents an important first step towards the realization of large planar devices, since we demonstrated that this technology can be successfully scaled up by using partially overlapping laser spots. It has been proved that multiple laser pulses, up to four, do not jeopardize the high purity of the germanium material, the depth distribution and the activation of charge carriers in the junction, nor the barrier height in the $\mathrm{n}+$ contact, the latter being particularly pronounced as highlighted by measurements of the reverse leakage current. I-V measurements showed the good electrical insulation between both segments and guard ring obtained by the described photolithographic method.

One prototype was also subjected to a typical annealing treatment performed to remove the radiation damage of complex detectors and it was found that the detector performance and the germanium purity are completely preserved. this technology to improve the present performance of complex, position sensitive and highly segmented HPGe detectors employed by modern gamma-ray tracking spectrometers. Considering that these detectors are not planar, the next important step will be the application of this technology to surfaces with different crystal orientations and different geometries (curved surfaces).

Acknowledgements This project has received funding from the European Union's Horizon 2020 research and innovation programme under grant agreement n. 654002 .

Funding Open access funding provided by Università degli Studi di Padova within the CRUI-CARE Agreement.

Data Availability Statement This manuscript has no associated data or the data will not be deposited. [Authors' comment: The datasets generated during and/or analysed during the current study are available from the corresponding author on reasonable request.]

Open Access This article is licensed under a Creative Commons Attribution 4.0 International License, which permits use, sharing, adaptation, 
distribution and reproduction in any medium or format, as long as you give appropriate credit to the original author(s) and the source, provide a link to the Creative Commons licence, and indicate if changes were made. The images or other third party material in this article are included in the article's Creative Commons licence, unless indicated otherwise in a credit line to the material. If material is not included in the article's Creative Commons licence and your intended use is not permitted by statutory regulation or exceeds the permitted use, you will need to obtain permission directly from the copyright holder. To view a copy of this licence, visit http://creativecomm ons.org/licenses/by/4.0/.

\section{References}

1. J. Simpson, The AGATA project. J. Phys. G 31, S1801-S1806 (2005)

2. M.A. Deleplanque, I.Y. Lee, K. Vetter, G.J. Schmid, F.S. Stephens, R.M. Clark, R.M. Diamond, P. Fallon, A.O. Macchiavelli, Nucl. Inst. Methods A 430, 292 (1999)

3. W.H. Berninger, IEEE Trans. Nucl. Sci. 21(1), 374-378 (1974)

4. P.N. Luke, IEEE Trans. Nucl. Sci. NS 31, 312 (1984)

5. YuB Gurov et al., Instrum. Exp. Technol. 50, 757 (2007)

6. G.S. King III, F.T. Avignone III, C.E. Cox, T.W. Hossbach, W. Jennings, J.H. Reeves, Nucl. Instrum. Methods Phys. Res. A 595, $599(2008)$

7. W.L. Hansen, E.E. Haller, IEEE Trans. Nucl. Sci. NS 24, 61 (1977)

8. P.N. Luke, C.P. Cork, N.W. Madden, C.S. Rossington, M.F. Wesela, IEEE Trans. Nucl. Sci. NS 39, 590 (1992)

9. M. Amman, P.N. Luke, S.E. Boggs, Nucl. Instrum. Methods Phys. Res. A 579, 886 (2007)

10. Q. Looker, Fabrication Process Development for High-Purity Germanium Radiation Detectors with Amorphous Semiconductor Contacts (University of California, Berkeley, 2014). PhD Thesis

11. Q. Looker, M. Amman, K. Vetter, Nucl. Instrum. Methods Phys. Res. A 777, 138 (2015)

12. E.L. Hull, R.H. Pehl, J.R. Lathrop, B.E. Suttle, Nucl. Instrum. Methods Phys. Res. A 626-627, 39 (2011)

13. E.G. Jackson, E.L. Hull, C.J. Lister, R.H. Pehl, Nucl. Instrum. Methods Phys. Res. A 774, 34 (2015)

14. B. Bruyneel, B. Birkenbach, J. Eberth, H. Hess, Gh Pascovici, P. Reiter, A. Wiens, D. Bazzacco, E. Farnea, C. Michelagnoli, F. Recchia, Eur. Phys. J. A 49, 61 (2013)

15. R. H. Pehl, et al. IEEE Trans. Nucl. Sci. NS-26, 321 (1979)
16. N. Abgrall, et al., in AIP Conference Proceedings Volume 1894, 16 October 2017, Article number 020027

17. K.J. Kang et al., Front. Phys. 8(4), 412-437 (2013)

18. D.H. Lowndes, T.W. Raudorf, Nucl. Instrum. Methods Phys. Res. A 240, 362 (1985)

19. C. Carraro, R. Milazzo, F. Sgarbossa, D. Fontana, G. Maggioni, W. Raniero, D. Scarpa, L. Baldassarre, M. Ortolani, A. Andrighetto, D.R. Napoli, D. De Salvador, E. Napolitani, Appl. Surf. Sci. 509, 145229 (2020)

20. G. Maggioni, S. Carturan, W. Raniero, S. Riccetto, F. Sgarbossa, V. Boldrini, R. Milazzo, D.R. Napoli, D. Scarpa, A. Andrighetto, E. Napolitani, D. De Salvador, Eur. Phys. J. A 54, 34 (2018)

21. V. Boldrini, G. Maggioni, S. Carturan, W. Raniero, F. Sgarbossa, R. Milazzo, D.R. Napoli, E. Napolitani, R. Camattari, D. De Salvador, J. Phys. D Appl. Phys. 52, 035104 (2019)

22. E.L. Hull, E. Jackson, C.J. Lister, R.H. Pehl, Nucl. Instrum. Methods Phys. Res. A 762, 125-129 (2014)

23. E. Jackson, E.L. Hull, C.J. Lister, R.H. Pehl, Nucl. Instrum. Methods Phys. Res. A 774, 34-41 (2015)

24. S. Brotzmann, H. Bracht, J. Appl. Phys. 103, 033508 (2008)

25. G. Maggioni, D.R. Napoli, J. Eberth, M. Gelain, S. Carturan, M.G. Grimaldi, S. Tatì, Eur. Phys. J. A 51, 141 (2015)

26. S. Carturan, G. Maggioni, S.J. Rezvani, R. Gunnella, N. Pinto, M. Gelain, D.R. Napoli, Mater. Chem. Phys. 161, 116 (2015)

27. L.J. Van der Pauw, Philips Res. Rep. 13, 1-9 (1958)

28. R.L. Petritz, L. Richard, Phys. Rev. 110, 1254-1262 (1958). https:// doi.org/10.1103/PhysRev.110.1254

29. R. Baron, G.A. Shifrin, O.J. Marsh, J.W. Mayer, J. Appl. Phys. 40, 3702 (1969). https://doi.org/10.1063/1.1658260

30. J. Llacer, Nucl. Instrum. Methods 98, 259 (1972)

31. C. Claeys, E. Simoen, Germanium-Based Technologies (Elsevier, Amsterdam, 2007)

32. Yu. B. Gurov, V. Karpukhin, S. Rozov, V. Sandukovsky, D. Borowicz, J. Kwiatkowska, B. Rajchel, J. Yurkowski, Instrum. Exp. Tech. 52, 137-140 (2009)

33. G. Maggioni, D.R. Napoli, J. Eberth, M. Gelain, S. Carturan, M.G. Grimaldi, S. Tatì, Eur. Phys. J. A 51, 141 (2015)

34. https://www.ikp.uni-koeln.de/research/agata/talks/AGATA_ GSI2012-Reiter.pptx. Accessed 7 Apr 2021

35. W. Coburn, S.E. Boggs, S. Amrose, R.P. Lin, M.T. Burks, M. Amman, P.N. Luke, N.W. Madden, E.L. Hull, in 2001 IEEE Nuclear Science Symposium Conference Record (Cat. No.01CH37310), San Diego, CA, USA, 2001, vol. 1, pp. 226-229 